\title{
IncRNA KCNQIOTI enhances the
}

\section{chemoresistance of oxaliplatin in colon cancer by targeting the miR-34a/ATG4B pathway}

This article was published in the following Dove Medical Press journal: OncoTargets and Therapy

\author{
Yongchao $\mathrm{Li}^{\prime}$ \\ Changfeng $\mathrm{Li}^{2}$ \\ Dandan $\mathrm{Li}^{2}$ \\ Lei Yang ${ }^{2}$ \\ Jingpeng Jin ${ }^{2}$ \\ Bin Zhang ${ }^{2}$ \\ 'Department of Gastrointestinal \\ Surgery, China-Japan Union Hospital \\ of Jilin University, Changchun, Jilin \\ 1 30033, China; ${ }^{2}$ Department of \\ Endoscopy Center, China-Japan \\ Union Hospital of Jilin University, \\ Changchun, Jilin I30033, China
}

Purpose: The chemoresistance of colon cancer to oxaliplatin (L-OHP) indicates poor prognosis. Long non-coding RNA (lncRNA) KCNQ1OT1 (KCNQ1 opposite strand/antisense transcript 1) has been shown to participate in the tumorigenesis of several types of cancers. However, little is known about the role of KCNQ1OT1 in the chemoresistance and prognosis of colon cancer. Materials and methods: Quantitative-PCR and Western blot were used to measure the expression profiles of KCNQ1OT1, miR-34a, and Atg4B in colon cancer tissues and cells. Cell viability assay and flow cytometry were used to examine their effects on cell proliferation and death. Cleavage of LC3 and GFP-LC3 plasmid transfection were used to detect autophagic activity. Double luciferase reporter assay was used to verify the interactions between miRNA and lncRNA or mRNA. Xenograft tumor model was used to verify the effects of KCNQ1OT1 in vivo. Results: In this study, it is shown that the expression level of KCNQ1OT1 was increased in tumor, which indicated poor prognosis in colon cancer patients. Using colon cancer cell lines HCT116 and SW480, it was demonstrated that knockdown of KCNQ1OT1 decreased the cell viability and increased the apoptosis rates upon L-OHP treatment. Further studies indicated that Atg4B upregulation was partially responsible for KCNQ1OT1-induced protective autophagy and chemoresistance. Moreover, miR-34a functioned as a bridge between KCNQ1OT1 and Atg4B, which could be sponged by KCNQ1OT1, while it could also bind to the 3'-UTR of Atg4B and downregulate its expressions. Finally, we show that the KCNQ1OT1/miR-34a/Atg4B axis regulated the chemoresistance of colon cancer cells in vitro and in vivo.

Conclusion: IncRNA KCNQ1OT1 promoted the chemoresistance of colon cancer by sponging miR-34a, thus upregulating the expressions of Atg4B and enhancing protective autophagy. KCNQ1OT1 might become a promising target for colon cancer therapeutics.

Keywords: KCNQ1OT1, colorectal cancer, protective autophagy, miR-34a, Atg4B, chemoresistance

\section{Introduction}

Colorectal cancer is the third prevalent type of cancer and accounts for one-tenth of all cancer-related deaths in human. ${ }^{1}$ Especially in China, more than 376,300 new cases and 191,000 deaths occurred in 2015 due to colorectal cancer. ${ }^{2}$ Oxaliplatin (L-OHP) is a first-line chemotherapy for colon cancer, which functions by covalently binding to DNA and forming platinum-DNA adducts to inhibit DNA replication and transcription. ${ }^{3}$ However, failure of cancer therapy is due to drug resistance, which brings poor prognosis for these patients. ${ }^{4}$

Inducing apoptosis is one of the most important mechanisms for the chemotherapy of L-OHP. Meanwhile, cancer cells would adopt autophagy as a protective response toward chemotherapy. ${ }^{5}$ Generally, autophagy promotes tumor cell survival and
Correspondence: Changfeng $\mathrm{Li}$ Department of Endoscopy Center, China-Japan Union Hospital of Jilin University, No. 126, Xiantai Street, Changchun, Jilin I30033, China Tel +86431 84641026 Email changfengli1975@I63.com hereby accept the Terms. Non-commercial uses of the work are permitted without any furcher permission from Dove Medical Press Limited, provided the work is properly attributed. For permission for commercial use of this work, please see paragraphs 4.2 and 5 of our Terms (https://www.dovepress.com/terms.php). 
decreases sensitivity to chemotherapy. ${ }^{5,6}$ Therefore, targeting autophagy has become an important strategy to improve the chemoresistance in colon cancer therapies. ${ }^{7}$ Long non-coding RNAs (lncRNAs) are a group of non-coding RNAs longer than 200 nucleotides. ${ }^{8,9}$ Recently, lncRNA has been found to function as decoys to sponge miRNAs, thus decreasing the expression levels of certain miRNAs, which is also an important mechanism of lncRNA-regulated gene function. ${ }^{10,11}$ IncRNA KCNQ1OT1 (KCNQ1 opposite strand/antisense transcript 1) has been demonstrated to be a chromatin regulatory RNA. ${ }^{12,13}$ Recent studies also revealed that KCNQ1OT1 participated in tumorigenesis and chemoresistance of several types of cancers. ${ }^{14,15}$ However, the role of KCNQ1OT1 in colon cancer and whether KCNQ1OT1 participates in the chemoresistance of L-OHP have not been elucidated.

\section{Materials and methods}

\section{Patients and ethics statement}

The protocols for using the colon cancer samples and analyzing patient data were approved by the ethics committee of the China-Japan Union Hospital of Jilin University. This study was conducted in accordance with the Declaration of Helsinki. The colon cancer patients were diagnosed in our hospital, who underwent surgeries and treatments in our hospital. Written informed consent was provided by each enrolled patient if he/she was still alive or by his/her first-degree relative if he/she had died. Tumor and adjacent tissues were snap-frozen in liquid nitrogen immediately after extraction and stored at $-80^{\circ} \mathrm{C}$.

\section{Cell culture and reagents}

Human colon cancer cell lines HCT116 and SW480 were purchased from American Type Culture Collection (ATCC, Manassas, VA, USA). The cells were cultured in DMEM supplemented with 10\% FBS, and antibiotics (penicillin and streptomycin) were added at the temperature of $37^{\circ} \mathrm{C}$ with $5 \% \mathrm{CO}_{2}$. The cell culture reagents were purchased from Thermo Fisher Scientific (Waltham, MA, USA). Chloroquine (CQ), 3-methyladenine (3-MA), and L-OHP were purchased from Sigma-Aldrich Co. (St Louis, MO, USA). Annexin-V and propidium iodide $(\mathrm{PI})$ dyes were purchased from $\mathrm{BD}$ Biosciences (San Jose, CA, USA). The specific siRNAs for KCNQ1OT1 and Atg4B and negative control were synthesized and purchased from Genecopoeia (Guangzhou, China). The miR-34a mimic, inhibitor, and their negative controls were synthesized and purchased from Gene Pharma (Shanghai, China).

\section{Quantitative reverse transcription PCR (qRT-PCR)}

The methods of extracting RNAs and conducting the qRT-PCR have been described previously. ${ }^{16}$ The primers used are listed in Table S1.

\section{Cell viability assay}

Cell viability assay was conducted using the Cell Counting Kit-8 (Dojindo, Kumamoto, Japan) as described previously. ${ }^{16}$

\section{Detection of apoptosis}

After treatments, colon cancer cells were digested with trypsin and washed with PBS twice. Then, the cells were incubated with Annexin-V and PI for $30 \mathrm{~min}$ at room temperature. Flow cytometry was performed, and the statistical diagrams were drawn by using FlowJo 7.5.

\section{Luciferase reporter assay}

Dual luciferase reporter assays were performed to verify the direct interactions between KCNQ1OT1 and miR-34a and miR-34a and the $3^{\prime}$-UTR of Atg4B mRNA. PCR was conducted using the PrimeSTAR DNA polymerase (Takara, Kusatsu, Japan) to amplify the KCNQ1OT1 cDNA containing the predicted miR-34a binding site and the $3^{\prime}$-UTR of Atg4B cDNA containing the predicted miR-34a binding site. The primers used are presented in Table S2. Then, the PCR products were purified and cloned to the pmirGLO vector. Afterward, we co-transfected the pmirGLO-WTKCN1 or -Mut-KCN1 with miR-34a mimics or miRNA-NC into colon cancer cells with Lipofectamine 3000. Also, the pmirGLO-WT-Atg4B or -Mut-Atg4B was transfected in a similar way. The luciferase activity was conducted after $48 \mathrm{~h}$ of transfection using the luciferase assay kit (Promega Corporation, Fitchburg, WI, USA) and the Promega GloMax 20/20 machine.

\section{Western blot}

Western blot was conducted as described previously. ${ }^{16,17}$ The primary antibodies used were as follows: anti-PARP (Abcam, Cambridge, MA, USA), anti-Atg4B (Abcam), antiLC3 (Sigma-Aldrich Co.), and anti-GAPDH (Santa Cruz Biotechnology Inc., Dallas, TX, USA).

\section{Animal experiments}

Male BALB/c nude mice (4-5 weeks old) were purchased from the Model Animal Research Center of Nanjing University and maintained in a specific pathogen-free facility. The protocol was approved by the Animal Ethics 
Committee of the China-Japan Union Hospital of Jilin University and was in accordance with the Guidelines for the welfare and use of animals in cancer research. ${ }^{18}$ To evaluate the in vivo chemoresistance of colon cancer cells to L-OHP, HCT116-siNC, HCT116-siKCNQ1OT1, SW480-siNC cells, and SW480-siKCNQ1OT1 were inoculated subcutaneously in the right flank of the nude mice. Tumors were measured every 7 days, and tumor volume was calculated. Five weeks after inoculation, the mice were killed, and the tumors were collected for further experiments.

\section{Statistical analysis}

The data are presented as mean \pm SEM. For the comparisons of mean values between two groups, unpaired Student's $t$-test was used to examine the differences. For the comparisons of mean values among three or more groups, ANOVA followed by Bonferroni post hoc tests were used to examine the differences. Survival data of patients were evaluated by the Kaplan-Meier survival curves, and the log-rank test was used to evaluate the differences between groups. The survival data were evaluated using univariate and multivariate Cox's proportional hazards model to determine the association between the risk factors and clinical outcomes, with the HR and 95\% CIs calculated. All the data analysis was conducted using GraphPad Software, Inc. (La Jolla, CA, USA) or SPSS 19.0 (IBM Corporation, Armonk, NY, USA). Two-sided $P$-value $<0.05$ was considered to be statistically significant.

\section{Results}

\section{Upregulation of KCNQIOTI is correlated with unfavorable prognosis in colon cancer}

First, qRT-PCR analysis was performed to detect the expression profiles of lncRNA KCNQ1OT1 in tissue samples of 45 clinical colon cancer cases. It is shown in Figure 1A that the expression levels of KCNQ1OT1 were significantly increased in tumor compared with adjacent normal tissues. Moreover, it is shown that in the tissue samples of stage I and II patients, the expression levels of KCNQ1OT1 were lower than those of stage III and IV patients (Figure 1B). Also, we used the Cox's proportional hazards analysis to analyze the prognosis of colon cancer patients with the risk factors (Table 1). It is shown that, besides tumor stages, the KCNQ1OT1 expression levels were correlated with the poor prognosis of colon cancer patients using the multivariate analysis. In Figure $1 \mathrm{C}$, we divided the patients into low $(\mathrm{N}=25)$ and high expression groups $(\mathrm{N}=20)$. Then, to investigate the clinical significance of KCNQ1OT1 in colon cancer, the correlations between its expression pattern and clinicopathological characteristics were analyzed. Data in Table 2 show that the expression levels of KCNQ1OT1 were significantly correlated with the tumor size $(P=0.036)$, tumor stage $(P=0.003)$, and the tumor stage $(P=0.003)$, whereas insignificantly correlated with gender, age, and distant metastasis. Furthermore, using Kaplan-Meier survival analysis, it is shown that high expression of KCNQ1OT1 was associated with poor overall survival of colon cancer patients $(P<0.05$, Figure 1D), suggesting that patients with higher levels of KCNQ1OT1 might be less sensitive to chemotherapy treatments.

Therefore, to further identify the effects of KCNQ1OT1 on the sensitivity of colon cancer cells to L-OHP, we used two colon cancer cells lines, HCT116 and SW480. It is shown in Figure 1E that KCNQ1OT1 knockdown by specific siRNA decreased the cell viability of both HCT116 and SW480 cells, treated with different concentrations of L-OHP. Meanwhile, apoptosis rates were also measured by flow cytometry. It showed that KCNQ1OT1 knockdown significantly increased the apoptosis rates in both HCT116 and SW480 cells upon $10 \mu \mathrm{mol} / \mathrm{L}$ L-OHP treatment (Figure $1 \mathrm{~F}$ and $\mathrm{G}$ ). These results indicated that lncRNA KCNQ1OT1 plays important roles in the chemoresistance of colon cancer by regulating the cell death.

\section{KCNQIOTI promoted the protective autophagy by upregulating Atg4B expression in colon cancer cells}

To further identify the mechanisms of KCNQ1OT1-induced chemoresistance, we detected the levels of autophagy, which is one of the most common protective reactions upon outside stress. It is shown in Figure 2A that L-OHP induced a significant cleavage of LC3-II from LC3-I, whereas inhibition of autophagy by 3-MA or CQ promoted the cleavage of PARP, indicating enhanced apoptosis. Therefore, L-OHP treatment induced a protective autophagy in colon cancer cells. Furthermore, we investigated the expression levels of PARP and LC3-II/LC3-I upon KCNQ1OT1 knockdown. Figure 2B shows that siKCNQ1OT1 transfection upregulated the cleavage of PARP whereas inhibited the cleavage of LC3-I to LC3-II, indicating that KCNQ1OT1 regulated autophagy and therefore influencing the chemosensitivity of colon cancer cells to L-OHP. We measured the expression patterns of $A \operatorname{tg} 4 \mathrm{~B}$, which is one of the main enzymes responsible for the cleavage of $\mathrm{LC} 3$, thus promoting the 

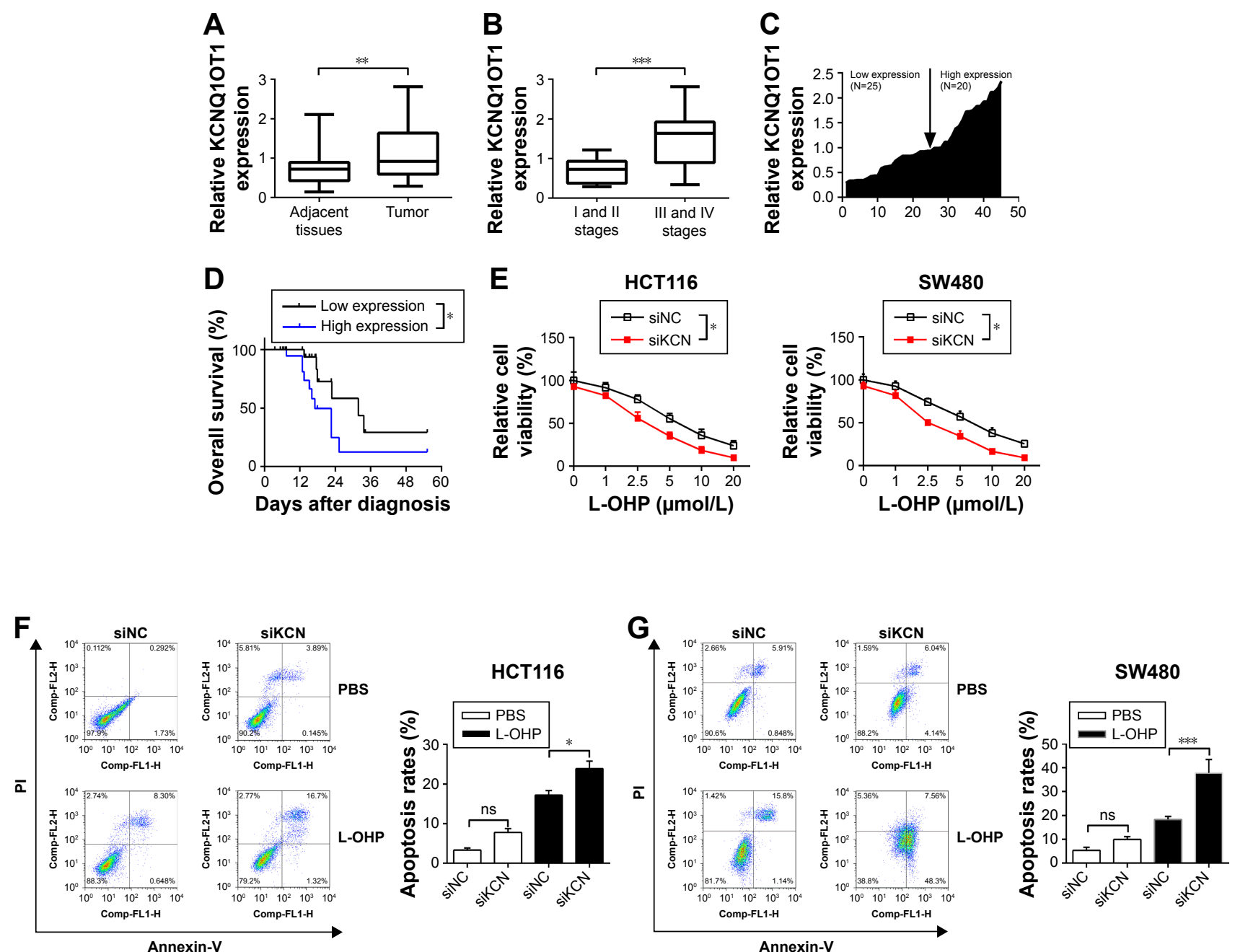

Figure I The expression profiles of IncRNA KCNQIOTI in colon cancer patients and the correlation with prognosis.

Notes: $(\mathbf{A})$ The expression levels of KCNQIOTI in colon cancer tissues and corresponding adjacent tissues were detected by $\mathrm{qRT}$-PCR. $* * P<0.0 \mathrm{I}$. (B) The expression levels of KCNQIOTI in the tumor tissues of stage I/II and stage III/IV patients. $* * * P<0.000 \mathrm{I}$. (C) The fold changes of KCNQIOTI expression level in each patient were measured. (D) Kaplan-Meier curves of overall survival and log-rank test of patients with low and high KCNQIOTI expression levels. $* P<0.05$. (E) HCTII6 and SW480 cells were transfected with KCNQIOTI siRNA and negative control, and then the cell viability assay was conducted with different concentrations of L-OHP. $* P<0.05$. (F and $\mathbf{G})$ The apoptosis rates of colon cancer cells were measured by flow cytometry. $* P<0.05$ and $* * * P<0.000$ I. Data are presented as mean $\pm S E M$.

Abbreviations: L-OHP, oxaliplatin; IncRNA, long non-coding RNA; PI, propidium iodide; qRT-PCR, quantitative real-time PCR; ns, not significant.

formation of autophagosome. ${ }^{19}$ It showed that KCNQ1OT1 knockdown unexpectedly downregulated the expression of Atg4B in both HCT116 and SW480 cells. In addition, we show that KCNQ1OT1 knockdown significantly decreases the number of cells with GFP-LC3 green fluorescence punctate (Figure 2C).

To further verify the relationship between KCNQ1OT1 and $\mathrm{Atg} 4 \mathrm{~B}$ in the chemoresistance of colon cancer, it is

Table I The Cox's proportional hazards analysis of the overall survival in colon cancer patients

\begin{tabular}{|c|c|c|c|c|c|c|}
\hline \multirow[t]{2}{*}{ Characteristics } & \multicolumn{3}{|c|}{ Univariate analysis } & \multicolumn{3}{|c|}{ Multivariate analysis } \\
\hline & HRs & $95 \% \mathrm{Cl}$ & $P$-values & HRs & $95 \% \mathrm{Cl}$ & $P$-values \\
\hline Gender & 1.068 & $0.416,2.746$ & 0.891 & 0.570 & $0.134,2.428$ & 0.448 \\
\hline Age (years) & 0.961 & $0.892,1.036$ & 0.301 & 1.020 & $0.919,1.133$ & 0.708 \\
\hline Tumor size & 1.569 & $1.158,2.126$ & $0.004 * *$ & 1.551 & $0.563,4.272$ & 0.396 \\
\hline Tumor stage (Dukes) & 0.245 & $0.072,0.835$ & $0.025^{*}$ & 0.004 & $0.000,0.255$ & $0.009 * *$ \\
\hline Lymphatic metastasis & 0.360 & $0.139,0.934$ & $0.036 *$ & 55.899 & $0.707,4,422.065$ & 0.071 \\
\hline Distant metastasis & 0.276 & $0.087,0.873$ & $0.028^{*}$ & 5.365 & $0.285,100.896$ & 0.262 \\
\hline KCNQIOTI expression levels & 6.056 & $2.678,13.694$ & $0.000 * * *$ & 5.581 & I.173, 26.562 & $0.03 I^{*}$ \\
\hline
\end{tabular}

Note: $* P<0.05, * * P<0.01$, and $* * * P<0.0001$. 
Table 2 Correlation between KCNQIOTI expression and clinicopathological features of colon cancer patients

\begin{tabular}{|c|c|c|c|}
\hline Characteristics & $\begin{array}{l}\text { Low } \\
\text { KCNQIOT I } \\
(\mathbf{N}=25)\end{array}$ & $\begin{array}{l}\text { High } \\
\text { KCNQ IOTI } \\
(\mathbf{N}=\mathbf{2 0})\end{array}$ & $P$-values \\
\hline Gender & & & 0.216 \\
\hline Male & 7 & 10 & \\
\hline Female & 18 & 10 & \\
\hline Age (years) & & & 0.50 \\
\hline$\geq 50$ & 20 & 14 & \\
\hline$<50$ & 5 & 6 & \\
\hline Tumor size $(\mathrm{cm})$ & & & $0.036 *$ \\
\hline$<3$ & 16 & 6 & \\
\hline$\geq 3$ & 9 & 14 & \\
\hline Tumor stage (Dukes) & & & $0.003 *$ \\
\hline I, II & 18 & 5 & \\
\hline III, IV & 7 & 15 & \\
\hline $\begin{array}{l}\text { Lymphatic metastasis } \\
\text { (yes) }\end{array}$ & 7 & 15 & $0.003 *$ \\
\hline $\begin{array}{l}\text { Distant metastasis } \\
\text { (yes) }\end{array}$ & 3 & 5 & 0.435 \\
\hline
\end{tabular}

Note: $* P<0.05$.

shown in Figure 2D that KCNQ1OT1 knockdown decreased viable cells, whereas co-transfection with Atg4B overexpression plasmid partially attenuated the proliferation inhibition, compared with the pcDNA vector plasmid in both HCT116 and SW480 cells. Meanwhile, the increased cell apoptosis, induced by KCNQ1OT1 knockdown, could also be partially reversed by Atg4B overexpression, compared with the vector group in Figure 2E. These results suggested that Atg4B played important roles in KCNQ1OT1induced protective autophagy and chemoresistance.

\section{MiR-34a is the connection between $\mathrm{KCNQ}$ IOTI and Atg4B regulating} chemoresistance in colon cancer cells

Next, we will investigate the mechanisms of KCNQ1OT1 in regulation of $\mathrm{Atg} 4 \mathrm{~B}$, as KCNQ1OT1 is a lncRNA that could function as a competing endogenous RNA (ceRNA) and sponging miRNA, and thus affecting the translation of target genes. First, we searched the possible miRNAs that could bind to the $3^{\prime}$-UTR of ATG4B in the databases of mirBase, Targetscan, and miRDB. It showed that miR-34a was discovered by all the three databases. Figure $3 \mathrm{~A}$ shows that miR-34a could bind to the $3^{\prime}$-UTR of Atg4B in the 266-273 nucleotides. To further verify the regulation of Atg4B, we transfected colon cancer cells with miR-34a mimic and miR-34a inhibitor. It showed that miR-34a mimic downregulated Atg4B expression levels whereas miR-34a inhibitor upregulated Atg4B expression (Figure 3B). In addition, luciferase reporter assay was used to further ascertain the binding affinity between miR-34a and Atg4B 3'-UTR. It showed that miR-34a mimic transfection could decrease the luciferase activity in the wild-type sequence, but not in the mutant sequence (Figure 3C).

Meanwhile, we investigated whether KCNQ1OT1 could also bind to miR-34a, thus sponging miR-34a. Using DIANALncBase software, ${ }^{20}$ we found two putative miR-34a binding sites with the highest binding scores (KCNQ1OT1-1 and KCNQ1OT1-2, Figure 3D). To guarantee that miR-34a is a functional target of KCNQ1OT1, we transfected KCNQ1OT1-specific siRNAs in colon cancer cells, showing that the expression levels of miR-34a were increased in both HCT116 and SW480 cells, compared with siNC (Figure 3E). Moreover, luciferase reporter assay was conducted to verify the binding affinity of the two binding sites in the sequence of KCNQ1OT1. It was demonstrated in Figure 3F that the two binding sites functioned in combination with the sequence of miR-34a, with high affinity to KCNQ1OT1-1. Since miR-34a functions as the bridge between KCNQ1OT1 and Atg4B, we then investigated whether miR-34a transfection would affect the chemosensitivity of colon cancer cells to L-OHP. It is shown in Figure $3 \mathrm{G}$ that transfection of miR-34a mimic decreased the cell viability while transfection of miR-34a inhibitor increased the cell viability upon L-OHP treatment, compared with miR-NC in both HCT116 and SW480 cells. As to the apoptosis rates, it is also shown in Figure $3 \mathrm{H}$ that miR-34 mimic transfection enhanced the apoptosis whereas miR-34a inhibitor transfection suppressed apoptosis in colon cancer cells. These results indicated that miR-34a functioned downstream of KCNQ1OT1 and regulated the expression of Atg4B. Then, we needed to further verify the existence and the role of KCNQ1OT1/miR-34a/Atg4B axis in the chemoresistance of colon cancer cells.

\section{KCNQIOTI/miR-34a/Atg4B axis regulated the chemoresistance of colon cancer cells in vitro and in vivo}

To further verify the existence and possible functions of KCNQ1OT1/miR-34a/Atg4B axis in colon cancer, we co-transfected KCNQ1OT1 siRNAs with miR-34a NC or inhibitor. It is shown in Figure 4A and B that upon transfection with KCNQ1OT1 siRNA to downregulate its expression levels, transfection with miR-34a inhibitor increased the cleavage of LC3, upregulated the expression of Atg4B, and suppressed the cleavage of PARP, compared with miR-NC, in both HCT116 and SW480 cells. Moreover, cell viability assay was performed, elucidating that the increased 
A
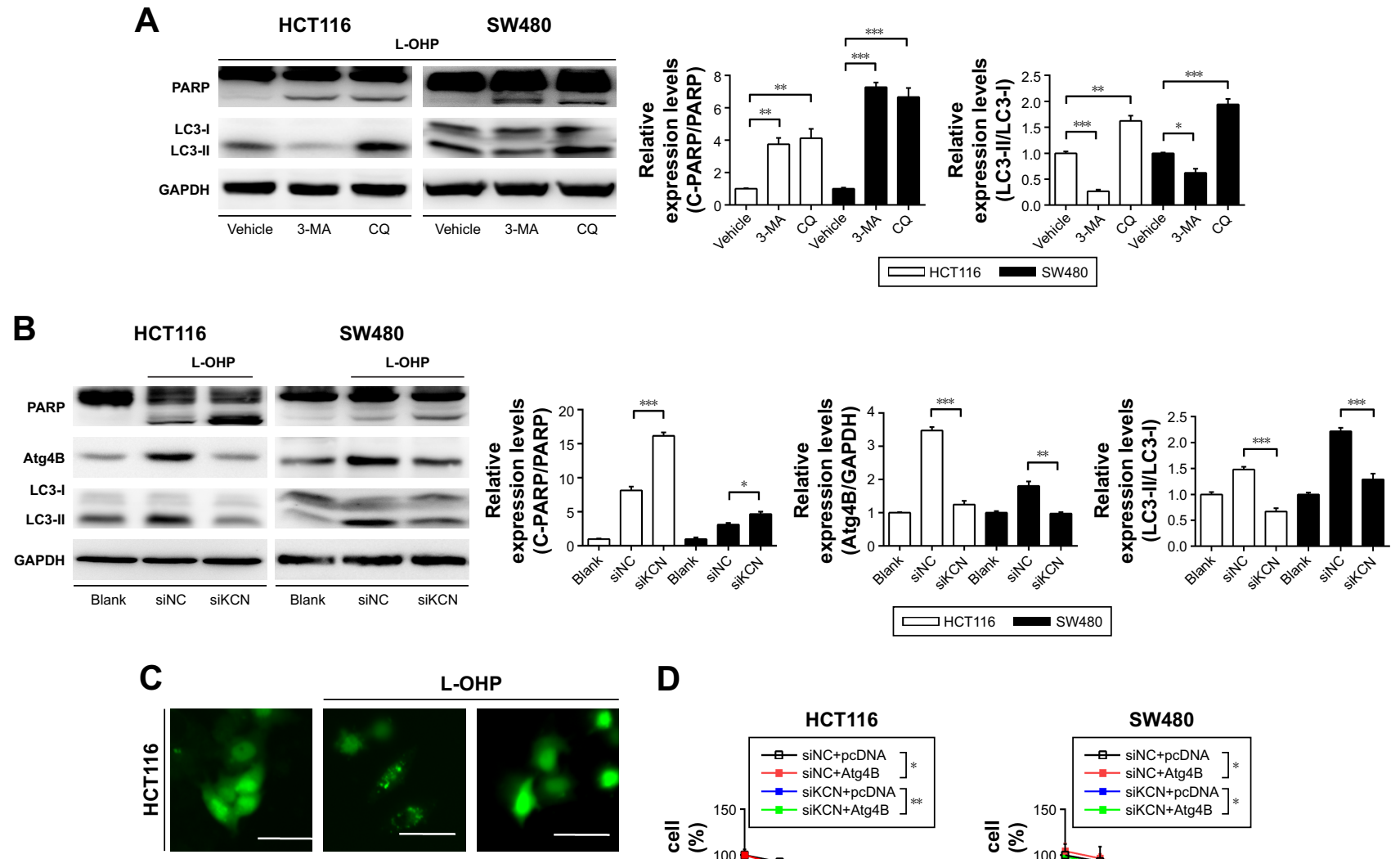

L-OHP
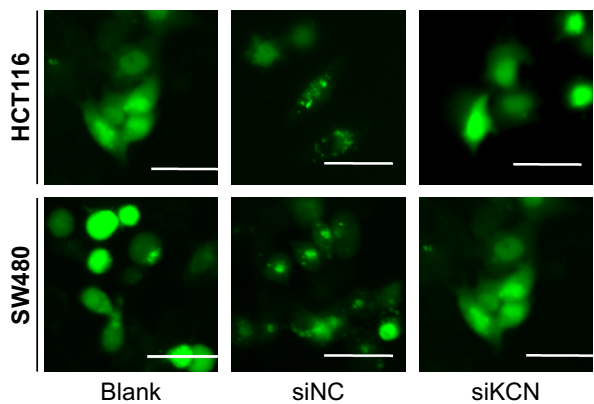

D
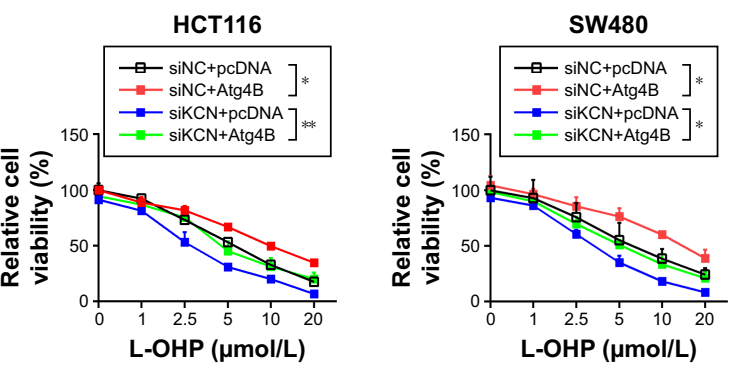

E
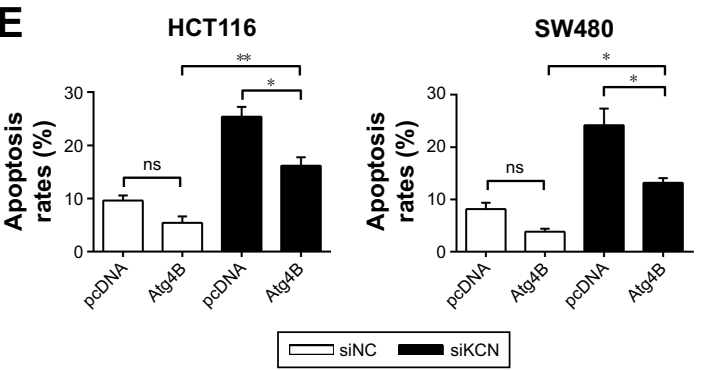

Figure 2 The effects of KCNQIOTI on L-OHP-induced protective autophagy and Atg4B expression levels in colon cancer cells.

Notes: (A) HCTII6 and SW480 cells were treated with L-OHP with the absence and presence of autophagy inhibitor 3-MA or CQ, and then the expression levels of PARP and LC3 were detected by Western blot, with GAPDH as a loading control. $* P<0.05$, $* * P<0.01$, and $* * * P<0.000$ I. (B) HCTII 6 and SW480 cells were transfected with siKCNQIOTI and treated with $10 \mu \mathrm{mol} / \mathrm{L}$ L-OHP for $24 \mathrm{~h}$, and then Western blot was performed. $* P<0.05$, $* * P<0.01$, and $* * * P<0.000 \mathrm{I}$. (C) HCTII6 and SW480 cells were treated with GFP-LC3 plasmid and then $10 \mu \mathrm{mol} / \mathrm{L} \mathrm{L-OHP}$ was added. After treatment, cells were observed in the fluorescence microscope. (D) HCTII6 and SW480 cells were co-transfected with siNC/siKCNQIOTI and pcDNA/pcDNA-Atg4B plasmids, and then cell viability assay was performed as above. $* P<0.0 \mathrm{I}$ and $* * P<0.0 \mathrm{I}$. (E) HCTII6 and SW480 cells were treated as shown in (D), and then the apoptosis rates were measured by flow cytometry. $* P<0.05$ and $* * P<0.01$. Abbreviations: CQ, chloroquine; L-OHP, oxaliplatin; 3-MA, 3-methyladenine; ns, not significant.

sensitivity of colon cancer cells to L-OHP by KCNQ1OT1 siRNA transfection was partially attenuated by the addition of miR-34a inhibitor (Figure 4C). In addition, we show that miR-34a transfection could also suppress the increased apoptosis rates upon KCNQ1OT1 knockdown (Figure 4D). These results indicated that KCNQ1OT1/miR-34a/Atg4B axis functioned in the chemoresistance of colon cancer cells to L-OHP in vitro.

Furthermore, we used the xenograft tumor model to demonstrate the role of KCNQ1OT1/miR-34a/Atg4B axis in chemoresistance of colon cancer in vivo. It showed that knockdown of KCNQ1OT1 in colon cancer cells significantly 
A

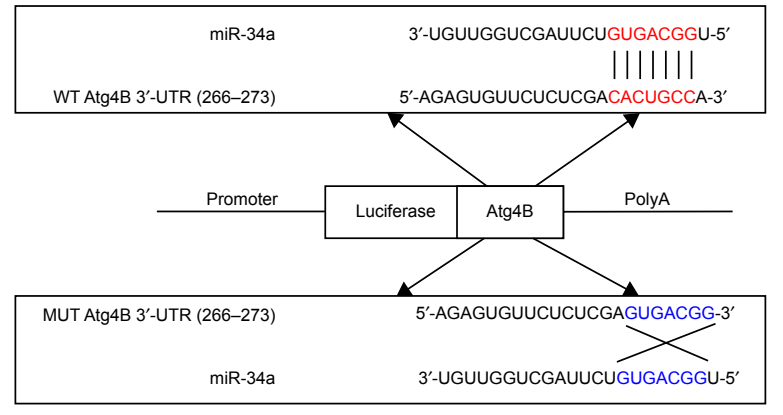

C

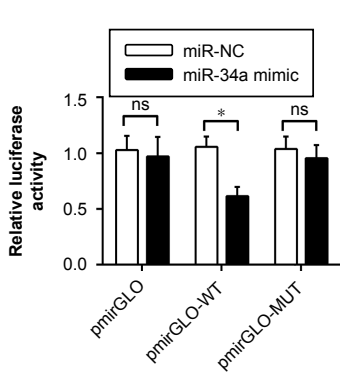

D

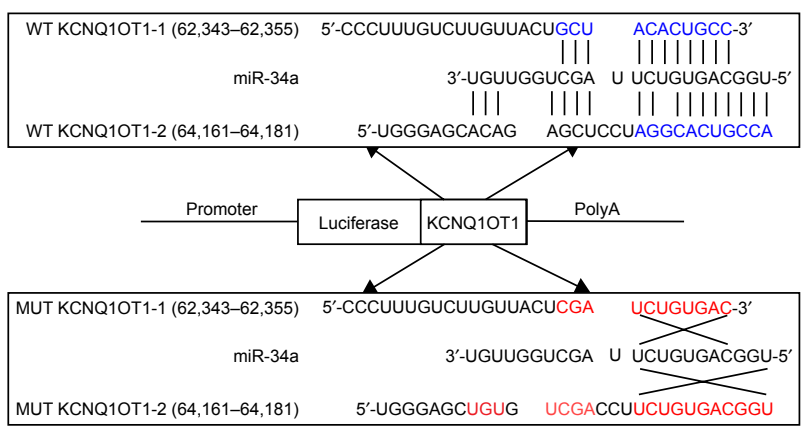

B

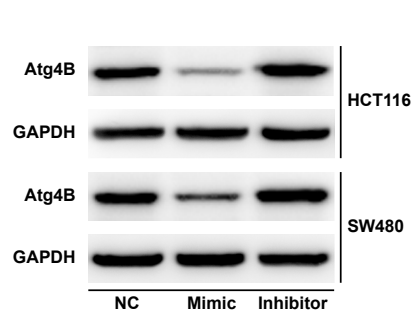

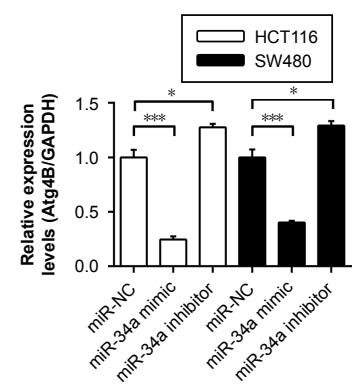

E

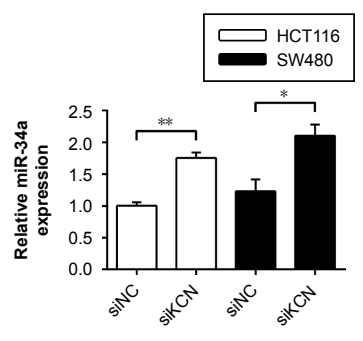

F

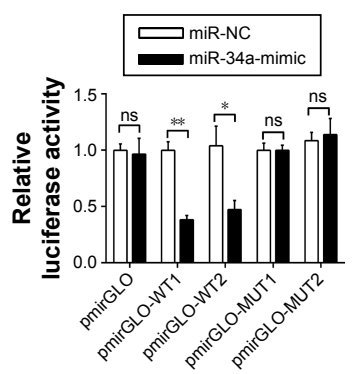

G

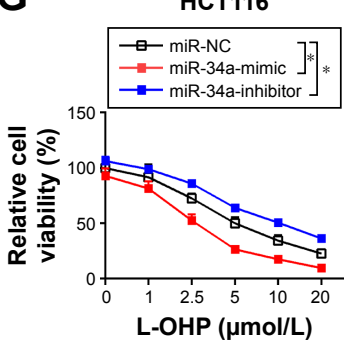

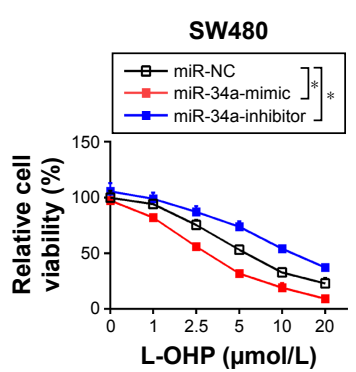

H

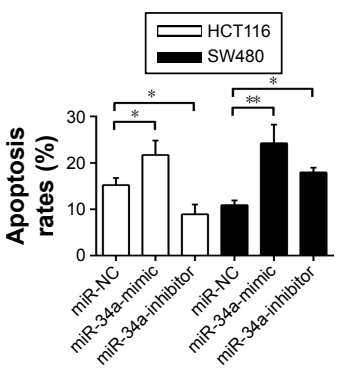

Figure 3 The mechanisms of miR-34a functioning as the connection between KCNQIOTI and Atg4B.

Notes: (A) Predicted binding site of miR-34a on the 3'-UTR of Atg4B mRNA base in the Targetscan database. (B) Western blot analysis was conducted to detect the expression levels of Atg4B, transfected with miR-34a mimic and NC. N=3, $* P<0.05$ and $* * * P<0.000$ I. (C) Double luciferase reporter assay results of the interaction between miR-34a and the $3^{\prime}-U T R$ of Atg4B mRNA. N=3, $* P<0.05$. (D) Predicted miR-34a binding sites in KCNQIOTI sequence based on the DIANA-LncBase analysis. (E) The expression level of miR-34a was measured by $q R T-P C R$ after being transfected with KCNQIOTI siRNA for 48 h. $N=3$, $* P<0.05$ and $* * P<0.01$. (F) The double luciferase reporter assay experiments investigating the binding affinity of miR-34a to KCNQIOTI. $* P<0.05$ and $* * P<0.01$. (G) Cell viability assay of miR-34a mimic or inhibitor on colon cancer cells. $\mathrm{N}=3$, $* P<0.05$. (H) $\mathrm{HCTI} 16$ and $\mathrm{SW} 480$ cells were treated as shown in $(\mathbf{G})$, and then apoptosis rates were measured using flow cytometry. $\mathrm{N}=3$, $* P<0.05$ and $* * P<0.01$.

Abbreviations: L-OHP, oxaliplatin; qRT-PCR, quantitative real-time PCR; ns, not significant.

decreased the xenograft tumor volume, compared with siNC, using both HCT116 and SW480 cells (Figure 4E). Meanwhile, the expression levels of miR-34a were increased upon KCNQ1OT1 knockdown in the xenograft tumor tissues (Figure 4F). The cleavage of PARP was increased whereas the cleavage of LC3 was decreased, with the downregulation of Atg4B upon L-OHP treatment (Figure 4G and H). These data further proved that KCNQ1OT1-mediated chemoresistance to L-OHP was due to sponging miR-34a and upregulation of Atg4B-induced autophagy.

\section{Discussion}

The chemoresistance of colon cancer to L-OHP treatment has been studied extensively. In this study, we show that lncRNA KCNQ1OT1 was upregulated in tumor tissues compared with adjacent tissues. High KCNQ1OT1 expression levels indicated poor prognosis in colon cancer patients. Furthermore, KCNQ1OT1 knockdown decreased the cell viability and increased the apoptotic cells upon L-OHP treatment. In addition, it was shown that KCNQ1OT1-induced protective autophagy by upregulating a key enzyme Atg4B. In detail, 
A

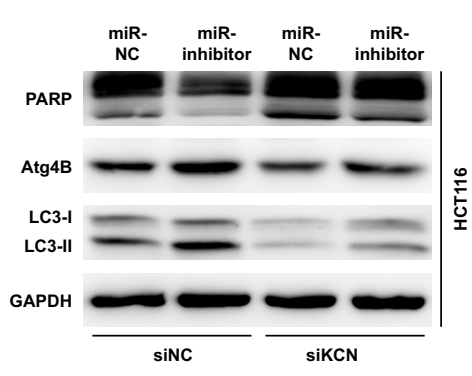

B

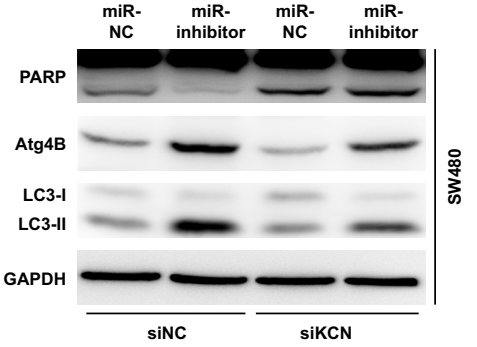

C

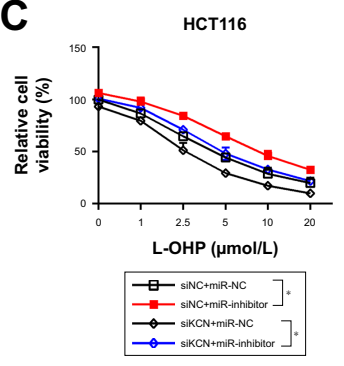

E

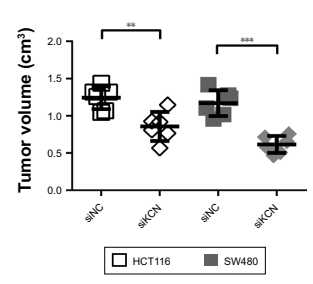

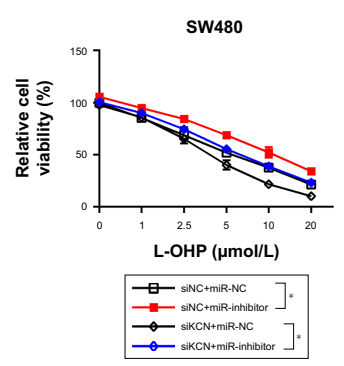

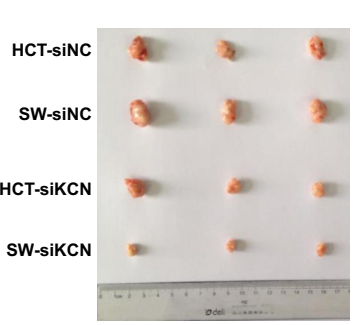

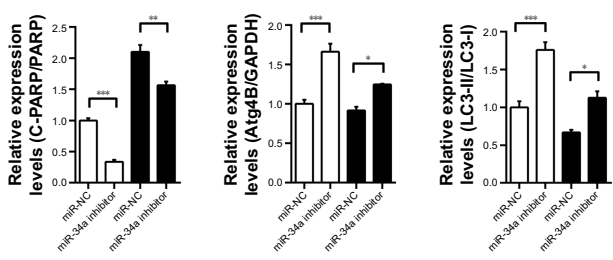
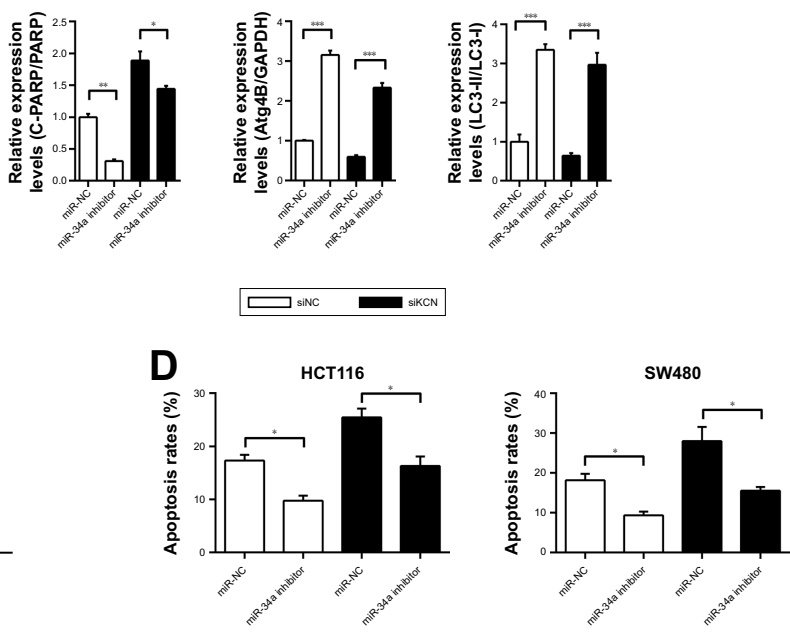

H
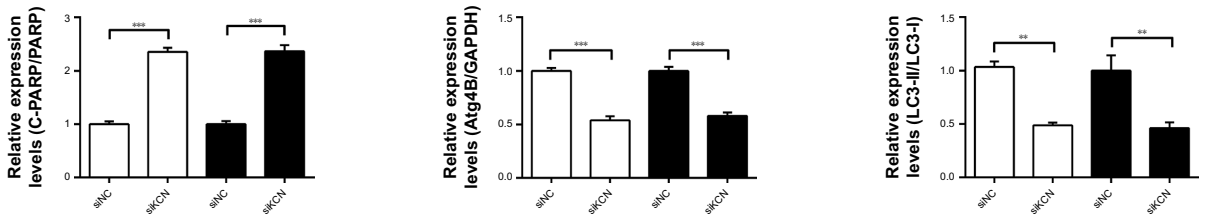

Figure 4 The effects of KCNQIOTI/miR-34a/Atg4B axis in regulating the sensitivity of colon cancer cells to L-OHP in vitro and in vivo.

Notes: (A and B) HCTII6 and SW480 cells were cotransfected with siNC/siKCNQIOTI and miR-34a NC/miR-34a inhibitors for 24 h, and then L-OHP was added and Western blot performed. $\mathrm{N}=3, * P<0.05, * * P<0.0 \mathrm{I}$, and $* * * P<0.000 \mathrm{I}$. (C and $\mathbf{D})$ Colon cancer cells were treated as shown in $(\mathbf{A})$, and then the cell viability and apoptosis rates were measured by CCK-8 and flow cytometry. ${ }^{*} P<0.05$. (E) HCTII6 and SW480 cells were pre-transfected with KCNQIOTI siRNA or siNC, and then the cells were injected subcutaneously to build the xenograft tumor model. $* * P<0.01$ and $* * * P<0.000 I$. (F) The expression levels of miR-34a were measured by $q R T-P C R$ in $x e n o g r a f t$ tumor. $* P<0.05$ and $* * P<0.0 I$. ( $\mathbf{G}$ and $\mathbf{H}$ ) The expression levels of PARP, Atg4B, and LC3 were detected by Western blot in the xenograft tumor tissues, and the bands were analyzed. $* * P<0.01$ and $* * * P<0.0001$.

Abbreviations: CCK-8, Cell Counting Kit-8; L-OHP, oxaliplatin; qRT-PCR, quantitative real-time PCR.

we demonstrated that miR-34a functioned as a bridge between KCNQ1OT1 and Atg4B, which could be sponged by KCNQ1OT1 and regulated the expression levels of Atg4B by binding to the $3^{\prime}$-UTR region. We then proved the existence of KCNQ1OT1/miR-34a/Atg4B axis in the chemoresistance of L-OHP in colon cancer both in vitro and in vivo.

Autophagy has been considered as an important cytoprotective process when cells experience outside stress, such 
as hypoxia, serum deprivation, toxicity, etc., to counteract apoptosis. Generally, autophagy promotes tumor cell survival and decreases sensitivity to chemotherapy. ${ }^{5}$ Inhibition of autophagy can sensitize tumors to both chemotherapy and radiotherapy. ${ }^{21}$ Similar to the Atg8 system in yeast, the C-terminal peptide of LC3 protein is cleaved by mammalian Atg4 homologs, namely Atg4A, Atg4B, Atg4C, and Atg4D in human. Atg4B is one of the human homologs of Atg4 enzymes that could cleave LC3 precursor to LC3-I; it has a glycine residue at its $\mathrm{C}$ terminus and resides in the cytosol. LC3-I could be further modified to LC3-II, which is thus promoting the formation of double-membrane autophagosome. Therefore, Atg4B functions as a key enzyme in the process of autophagy. ${ }^{22,23}$ Furthermore, Atg4B has been shown to participate in the chemoresistance in several cancer types. It has been shown that Atg4B functions in the chemoresistance of prostate cancer, lung cancer, and breast cancer cells. ${ }^{24-26}$ However, little is known about the role of Atg4B in colon cancer. In our study, we found that L-OHP-induced protective autophagy could be partially attenuated by Atg4B knockdown. miR-34a could bind to the 3'-UTR of Atg4B, thus increasing the sensitivity of colon cancer cells to L-OHP. In addition, the post-translational regulation of Atg4B has been studied widely recently. It has been shown that phosphorylation of ATG4B at Ser-383 and Ser-392 increases its hydrolase activity. ${ }^{27} \mathrm{Ni}$ et $\mathrm{al}^{28}$ elucidated that AKT-mediated phosphorylation of ATG4B enhances the Warburg effect in hepatocellular carcinoma cells. Therefore, elucidating the regulatory mechanisms of Atg4B might become a new therapeutic target for cancer chemotherapy.

Currently, lncRNAs have been shown to function in various cellular processes by multiple mechanisms. ${ }^{29}$ Moreover, sponging miRNA as decoys, also known as ceRNAs, ${ }^{29}$ has been studied recently as an important mechanism of lncRNA regulating gene expression. In our study, we show that lncRNA KCNQ1OT1 could sponge miR-34a, thus regulating the key enzyme Atg4B and the autophagic activities. lncRNA KCNQ1OT1 has been shown to be an imprinting gene that is associated with the occurrence of BeckwithWiedemann syndrome and related tumor development. ${ }^{12,30}$ Recently, KCNQ1OT1 has been shown to be linked with the tumorigenesis and chemoresistance. Zhang et $\mathrm{al}^{31}$ showed that KCNQ1OT1 regulated proliferation and cisplatin resistance in tongue cancer via miR-211-5p-mediated Ezrin/ Fak/Src signaling. Meanwhile, knockdown of lncRNA KCNQ1OT1 depressed chemoresistance to paclitaxel in lung adenocarcinoma. ${ }^{14}$ In our study, we found that KCNQ1OT1 expression levels were correlated with the prognosis of colon cancer patients. Moreover, we show that miR-34a is a new target of KCNQ1OT1, whereas KCNQ1OT1 could partially sensitize colon cancer cells to L-OHP treatment by inhibiting protective autophagy and enhancing apoptosis.

MiRNA-34a belongs to the miRNA-34 family, which controls the expression of a plethora of target proteins involved in cell cycle, differentiation, apoptosis, and antagonizes processes that are necessary for basic cancer cell viability and cancer stemness, metastasis, and chemoresistance. ${ }^{32,33}$ MiR-34a acts as a tumor suppressor in various cancer types, and emerging evidence have shown that miR-34a-based replacement therapy is a promising approach in cancer treatment. ${ }^{34-36}$ As to the role of miR-34a in cancer treatment, it has been shown that miR-34a functioned by antagonizing multidrug resistance of colon cancer. ${ }^{37}$ For example, in the 5-FU-resistant cell line DLD-1/5-FU, mature miR-34a transfection attenuated 5-FU resistance and inhibited growth in the parental cells. ${ }^{38} \mathrm{MiR}-34 \mathrm{a}$ decreased multidrug resistance and inhibited migration and invasion in colon cancer cells. ${ }^{39,40}$ However, in several studies, miR-34a has also been shown to function by enhancing chemoresistance. ${ }^{37}$ It was shown that inhibition of miR-34a sensitized docetaxel-resistant MCF-7 cells to the cytotoxic effects of docetaxel in drug-sensitive MCF-7 cells, which is correlated with the dysregulated cell cycle arrest. ${ }^{41}$ In another controversial study, miR-34a was downregulated in RPMI2650CR cell line and tumor tissues of patients with sinonasal squamous cell carcinomas, while a significant correlation existed between decreased miR-34a expression and poor prognosis. ${ }^{42}$ In our study, it was shown that miR-34a mimic transfection could enhance the chemosensitivity of colon cancer cells to L-OHP. Therefore, it is critical to fully understand the functional role of miR-34a, particularly its cancer-specific functions, through more mechanistic and detailed studies.

MiR-34a has also been shown as a prognostic marker in cancer patients. ${ }^{43-45}$ Several studies showed miR-34a as a tumor suppressor and indicated good prognosis. ${ }^{44,46}$ However, controversies also existed. It has been demonstrated that miR-34a overexpression indicated poor prognosis in colon cancer patients. ${ }^{47,48}$ Also, low levels of miR-34a are positive prognostic factors in human hepatocellular and advanced serous ovarian carcinoma. ${ }^{49}$ In our study, we did not further analyze the prognostic role of miR-34a in the survival results of colon cancer patients. Altogether, in our study, we found that IncRNA KCNQ1OT1 could directly bind to and sponge miR-34a, thus decreasing the levels of miR-34a and influencing the chemosensitivity of colon cancer cells to L-OHP. Therefore, we elucidated a new mechanism that the KCNQ1OT1/miR-34a/Atg4B axis modulated chemosensitivity of colon cancer to L-OHP; besides, targeting this 
axis might provide new insights into the chemotherapy of colon cancer.

\section{Conclusion}

IncRNA KCNQ1OT1 promoted the chemoresistance of colon cancer by sponging miR-34a, thus upregulating the expressions of $\mathrm{Atg} 4 \mathrm{~B}$ and enhancing protective autophagy. KCNQ1OT1 might become a promising target for colon cancer therapeutics.

\section{Disclosure}

The authors report no conflicts of interest in this work.

\section{References}

1. Siegel R, Desantis C, Jemal A. Colorectal cancer statistics, 2014. CA Cancer J Clin. 2014;64(2):104-117. doi:10.3322/caac.21220

2. Chen W, Zheng R, Baade PD, et al. Cancer statistics in China, 2015. CA Cancer J Clin. 2016;66(2):115-132. doi:10.3322/caac.21338

3. Kelland L. The resurgence of platinum-based cancer chemotherapy. Nat Rev Cancer. 2007;7(8):573-584. doi:10.1038/nrc2167

4. Martinez-Balibrea E, Martinez-Cardus A, Gines A, et al. Tumor-related molecular mechanisms of oxaliplatin resistance. Mol Cancer Ther. 2015;14(8):1767-1776. doi:10.1158/1535-7163.MCT-14-0636

5. Yang Z, Klionsky DJ. Eaten alive: a history of macroautophagy. Nat Cell Biol. 2010;12(9):814-822. doi:10.1038/ncb0910-814

6. Wu Y, Ni Z, Yan X, et al. Targeting the MIR34C-5p-ATG4B-autophagy axis enhances the sensitivity of cervical cancer cells to pirarubicin. Autophagy. 2016;12(7):1105-1117. doi:10.1080/15548627.2016. 1173798

7. Kumar A, Singh UK, Chaudhary A. Targeting autophagy to overcome drug resistance in cancer therapy. Future Med Chem. 2015;7(12): 1535-1542. doi:10.4155/fmc. 15.88

8. Quinn JJ, Chang HY. Unique features of long non-coding RNA biogenesis and function. Nat Rev Genet. 2016;17(1):47-62. doi:10.1038/ nrg. 2015.10

9. Nagano T, Fraser P. No-nonsense functions for long noncoding RNAs. Cell. 2011;145(2):178-181. doi:10.1016/j.cell.2011.03.014

10. Thomson DW, Dinger ME. Endogenous microRNA sponges: evidence and controversy. Nat Rev Genet. 2016;17(5):272-283. doi:10.1038/ nrg.2016.20

11. Kallen AN, Zhou XB, Xu J, et al. The imprinted H19 lncRNA antagonizes let-7 microRNAs. Mol Cell. 2013;52(1):101-112. doi:10.1016/j. molcel.2013.08.027

12. Korostowski L, Sedlak N, Engel N. The Kcnq1ot1 long non-coding RNA affects chromatin conformation and expression of Kenq1, but does not regulate its imprinting in the developing heart. PLoS Genet. 2012;8(9):e1002956. doi:10.1371/journal.pgen.1002956

13. Pandey RR, Mondal T, Mohammad F, et al. Kcnq1ot1 antisense noncoding RNA mediates lineage-specific transcriptional silencing through chromatin-level regulation. Mol Cell. 2008;32(2):232-246. doi:10.1016/j.molcel.2008.08.022

14. Ren K, Xu R, Huang J, Zhao J, Shi W. Knockdown of long non-coding RNA KCNQ1OT1 depressed chemoresistance to paclitaxel in lung adenocarcinoma. Cancer Chemother Pharmacol. 2017;80(2):243-250. doi:10.1007/s00280-017-3356-z

15. Li C, Miao R, Zhang J, Qu K, Liu C. Long non-coding RNA KCNQ1OT1 mediates the growth of hepatocellular carcinoma by functioning as a competing endogenous RNA of miR-504. Int J Oncol. 2018. doi: 10.3892/ijo.2018.4313. [Epub ahead of print]

16. Li C, Gao Y, Li Y, Ding D. TUG1 mediates methotrexate resistance in colorectal cancer via miR-186/CPEB2 axis. Biochem Biophys Res Commun. 2017;491(2):552-557. doi:10.1016/j.bbrc.2017.03.042
17. Li C, Zhang Y, Cheng X, et al. PINK1 and PARK2 suppress pancreatic tumorigenesis through control of mitochondrial iron-mediated immunometabolism. Dev Cell. 2018;46(4):441-455.e448. doi:10.1016/j. devcel.2018.07.012

18. Workman P, Aboagye EO, Balkwill F, et al. Guidelines for the welfare and use of animals in cancer research. Br J Cancer. 2010;102(11): 1555-1577. doi:10.1038/sj.bjc.6605642

19. Hemelaar J, Lelyveld VS, Kessler BM, Ploegh HL. A single protease, Apg4B, is specific for the autophagy-related ubiquitin-like proteins GATE-16, MAP1-LC3, GABARAP, and Apg8L. J Biol Chem. 2003; 278(51):51841-51850. doi:10.1074/jbc.M308762200

20. Paraskevopoulou MD, Vlachos IS, Karagkouni D, et al. DIANALncBase v2: indexing microRNA targets on non-coding transcripts. Nucleic Acids Res. 2016;44(D1):D231-D238. doi:10.1093/nar/gkv1270

21. Maiuri MC, Zalckvar E, Kimchi A, Kroemer G. Self-eating and selfkilling: crosstalk between autophagy and apoptosis. Nat Rev Mol Cell Biol. 2007;8(9):741-752. doi:10.1038/nrm2239

22. Sugawara K, Suzuki NN, Fujioka Y, Mizushima N, Ohsumi Y, Inagaki F. Structural basis for the specificity and catalysis of human Atg4B responsible for mammalian autophagy. J Biol Chem. 2005;280(48): 40058-40065. doi:10.1074/jbc.M509158200

23. Satoo K, Noda NN, Kumeta H, et al. The structure of Atg4B-LC3 complex reveals the mechanism of $\mathrm{LC} 3$ processing and delipidation during autophagy. EMBO J. 2009;28(9):1341-1350. doi:10.1038/emboj. 2009.80

24. Bortnik S, Choutka C, Horlings HM, et al. Identification of breast cancer cell subtypes sensitive to ATG4B inhibition. Oncotarget. 2016; 7(41):66970-66988. doi:10.18632/oncotarget.11408

25. Wu S, Su J, Qian H, Guo T. SLC27A4 regulate ATG4B activity and control reactions to chemotherapeutics-induced autophagy in human lung cancer cells. Tumour Biol. 2016;37(5):6943-6952. doi:10.1007/ s13277-015-4587-4

26. Liao H, Xiao Y, Hu Y, et al. Methylation-induced silencing of miR-34a enhances chemoresistance by directly upregulating ATG4B-induced autophagy through AMPK/mTOR pathway in prostate cancer. Oncol Rep. 2016;35(1):64-72. doi:10.3892/or.2015.4331

27. Yang Z, Wilkie-Grantham RP, Yanagi T, Shu CW, Matsuzawa S, Reed JC. ATG4B (Autophagin-1) phosphorylation modulates autophagy. J Biol Chem. 2015;290(44):26549-26561. doi:10.1074/jbc. M115.658088

28. Ni Z, He J, Wu Y, et al. AKT-mediated phosphorylation of ATG4B impairs mitochondrial activity and enhances the Warburg effect in hepatocellular carcinoma cells. Autophagy. 2018;14(4):685-701. doi: 10.1080/15548627.2017.1407887

29. Rafiee A, Riazi-Rad F, Havaskary M, Nuri F. Long noncoding RNAs: regulation, function and cancer. Biotechnol Genet Eng Rev. 2018;34(2):153-180.

30. Higashimoto K, Soejima H, Saito T, Okumura K, Mukai T. Imprinting disruption of the CDKN1C/KCNQ1OT1 domain: the molecular mechanisms causing Beckwith-Wiedemann syndrome and cancer. Cytogenet Genome Res. 2006;113(1-4):306-312. doi:10.1159/000090846

31. Zhang S, Ma H, Zhang D, et al. LncRNA KCNQ1OT1 regulates proliferation and cisplatin resistance in tongue cancer via miR-211-5p mediated Ezrin/Fak/Src signaling. Cell Death Dis. 2018;9(7):742. doi:10.1038/s41419-018-1111-y

32. Wu QB, Sheng X, Zhang N, Yang MW, Wang F. Role of microRNAs in the resistance of colorectal cancer to chemoradiotherapy. Mol Clin Oncol. 2018;8(4):528-532. doi:10.3892/mco.2018.1578

33. Misso G, Di Martino MT, De Rosa G, et al. Mir-34: a new weapon against cancer? Mol Ther Nucleic Acids. 2014;3:e194. doi:10.1038/ mtna. 2014.47

34. Li XJ, Ren ZJ, Tang JH. MicroRNA-34a: a potential therapeutic target in human cancer. Cell Death Dis. 2014;5:e1327. doi:10.1038/ cddis. 2014.270

35. Farooqi AA, Tabassum S, Ahmad A. MicroRNA-34a: a versatile regulator of myriads of targets in different cancers. Int J Mol Sci. 2017;18:10. doi:10.3390/ijms 18102089 
36. Beg MS, Brenner AJ, Sachdev J, et al. Phase I study of MRX34, a liposomal miR-34a mimic, administered twice weekly in patients with advanced solid tumors. Invest New Drugs. 2017;35(2):180-188. doi:10.1007/s10637-016-0407-y

37. Ghandadi M, Sahebkar A. MicroRNA-34a and its target genes: key factors in cancer multidrug resistance. Curr Pharm Des. 2016;22(7): 933-939.

38. Akao Y, Noguchi S, Iio A, Kojima K, Takagi T, Naoe T. Dysregulation of microRNA-34a expression causes drug-resistance to 5-FU in human colon cancer DLD-1 cells. Cancer Lett. 2011;300(2):197-204. doi:10.1016/j.canlet.2010.10.006

39. Lai M, Du G, Shi R, et al. MiR-34a inhibits migration and invasion by regulating the SIRT1/p53 pathway in human SW480 cells. Mol Med Rep. 2015;11(5):3301-3307. doi:10.3892/mmr.2015.3182

40. Li Y, Gong P, Hou JX, et al. miR-34a regulates multidrug resistance via positively modulating OAZ2 signaling in colon cancer cells. J Immunol Res. 2018;2018:7498514. doi:10.1155/2018/7498514

41. Kastl L, Brown I, Schofield AC. miRNA-34a is associated with docetaxel resistance in human breast cancer cells. Breast Cancer Res Treat. 2012;131(2):445-454. doi:10.1007/s10549-011-1424-3

42. Ogawa T, Saiki Y, Shiga K, et al. miR-34a is downregulated in cisdiamminedichloroplatinum treated sinonasal squamous cell carcinoma patients with poor prognosis. Cancer Sci. 2012;103(9):1737-1743. doi:10.1111/j.1349-7006.2012.02338.x
43. Ren F, Zhang X, Liang H, et al. Prognostic significance of MiR-34a in solid tumors: a systemic review and meta-analysis with 4030 patients. Int J Clin Exp Med. 2015;8(10):17377-17391.

44. Wang J, Dan G, Zhao J, et al. The predictive effect of overexpressed miR-34a on good survival of cancer patients: a systematic review and meta-analysis. Onco Targets Ther. 2015;8:2709-2719. doi:10.2147/ OTT.S84043

45. Zhang Y, Wei C, Guo CC, et al. Prognostic value of microRNAs in hepatocellular carcinoma: a meta-analysis. Oncotarget. 2017;8(63) 107237-107257. doi:10.18632/oncotarget.20883

46. Gao J, Li N, Dong Y, et al. miR-34a-5p suppresses colorectal cancer metastasis and predicts recurrence in patients with stage II/III colorectal cancer. Oncogene. 2015;34(31):4142-4152. doi:10.1038/onc.2014.348

47. Hiyoshi Y, Schetter AJ, Okayama H, et al. Increased microRNA-34b and-34c predominantly expressed in stromal tissues is associated with poor prognosis in human colon cancer. PLoS One. 2015;10(4):e0124899. doi:10.1371/journal.pone.0124899

48. Rapti SM, Kontos CK, Christodoulou S, Papadopoulos IN, Scorilas A. miR-34a overexpression predicts poor prognostic outcome in colorectal adenocarcinoma, independently of clinicopathological factors with established prognostic value. Clin Biochem . 2017;50(16-17):918-924. doi:10.1016/j.clinbiochem.2017.06.004

49. Agostini M, Knight RA. miR-34: from bench to bedside. Oncotarget. 2014;5(4):872-881. doi:10.18632/oncotarget.1825 


\section{Supplementary materials}

Table SI Primers used for qRT-PCR

\begin{tabular}{|c|c|c|}
\hline Genes & Forward $\left(5^{\prime}-3^{\prime}\right)$ & Reverse $\left(5^{\prime}-3^{\prime}\right)$ \\
\hline KCNQIOTI & АСТСАСТСАСТСАСТСАСТ & CTGGCTCСTTCTATCACATT \\
\hline GAPDH & TCTCTGCTCCTCCTGTTC & GTTGACTCCGACCTTCAC \\
\hline miR-34a & GTGTCTTAGCTGGTTGTTG & TACTTGCTGATTGCTTCCT \\
\hline U6 & GCTTCGGCAGCACATATACT & AACGCTTCACGAATTTGCGT \\
\hline
\end{tabular}

Abbreviation: qRT-PCR, quantitative real-time PCR.

Table S2 Primers used for luciferase reporter assay

\begin{tabular}{|c|c|c|}
\hline Genes & Forward $\left(5^{\prime}-3^{\prime}\right)$ & Reverse $\left(5^{\prime}-3^{\prime}\right)$ \\
\hline KCNQIOTI-I & CGGAATCCAGGAATTGAACTCAGC & GCTCTTGATTGCACTGTGGTCTG \\
\hline KCNQIOTI-2 & CGGAACTAGGAATCCAACTTACAAGG & GCTCTTGAAGAAAGTCATTGGTAGC \\
\hline Atg $4 B$ & CTAGCTACTCTGACCTACGACACT & CCGCTCGTTGAGGACGCTGAAGTA \\
\hline
\end{tabular}

\section{Publish your work in this journal}

OncoTargets and Therapy is an international, peer-reviewed, open access journal focusing on the pathological basis of all cancers, potential targets for therapy and treatment protocols employed to improve the management of cancer patients. The journal also focuses on the impact of management programs and new therapeutic agents and protocols on patient perspectives such as quality of life, adherence and satisfaction. The manuscript management system is completely online and includes a very quick and fair peer-review system, which is all easy to use. Visit http://www.dovepress.com/testimonials.php to read real quotes from published authors. 